\section{Street lighting for prevention of road traffic injuries}

Road traffic crashes are a major cause of death and injury, especially in low and middle income countries (LMICs). Over a million people die each year on the world's roads, and the number of injuries could be as high as 50 million. ${ }^{1}$ A systematic review was recently published on the Cochrane Database of Systematic Reviews, investigating the effects of street lighting on fatalities and injuries from road traffic crashes. ${ }^{2}$

Street lighting may improve a driver's visual capabilities and ability to detect roadway hazards, and can reduce contrast between headlight glare and the surrounding environment, preventing loss of visual clarity from contrast adaptation. However, street lighting may also adversely affect safety due to "risk compensation": drivers may "feel" safer and consequently might increase speed and reduce concentration. Previous systematic reviews ${ }^{34}$ disagreed about the level of evidence supporting the use of street lights, and this latest review aimed to provide a rigorous re-evaluation of the evidence.

The review sought studies of the effects of new or improved street lighting on crashes and injuries. No randomised controlled trials (RCTs) of street lighting were found, but the authors identified 17 controlled before-after studies (CBAs), which investigated the effect of street lighting on rural roads, trunk roads and motorways (interstate highways) in the USA, the UK, Germany and Australia. Twelve studies compared the installation of street lighting with a "before" period in which it was absent, four studies investigated the effect of improving existing street lighting, and one study investigated both of these phenomena in different areas. Ten studies looked at the effect of continuous (midblock) lighting along lengths of road, whereas six studies focused on non-continuous lighting, which occurs exclusively at junctions (intersections) or pedestrian crossings. One study examined both continuous and noncontinuous lighting. Ten studies collected data at the intervention site only, and in these cases reported daytime data were used in lieu of area control data, since street lighting was assumed to affect only night-time traffic. Seven studies utilised a control site separate from the intervention area, of which five provided usable control area data. The remaining two studies were treated as daytime controlled studies.

Risk of bias within individual studies was assessed in three ways. Firstly, the source of the outcome data was noted, to assess whether it was collected independently of the study. Secondly, authors investigated the matching of characteristics between roads on which the intervention was installed and control roads (such as size of roads or geographical area); and finally they recorded the length of time over which the data were collected. Risk of bias was judged to be low for the majority of the studies in the first two of these categories, although there was some potential for contamination of the control area (by lighting from the intervention area) in some studies. For the final category, risk of bias was unclear, although nearly all studies incorporated a data collection time of at least a year, thus accounting for seasonal daylight changes.

Authors pooled data from 15 of the 17 trials and concluded that when considered together, street lighting (whether new, improved, continuous or non-continuous) has a statistically significant effect on total crashes (pooled rate ratio (RR) 0.45 , $95 \%$ CI 0.29 to 0.69 for studies with area control data; pooled RR $0.68,95 \%$ CI 0.57 to 0.82 for studies with daytime control data), fatal crashes (pooled RR $0.34,95 \%$ CI 0.17 to 0.68 for studies with daytime control data; no area-controlled studies were included with this outcome), and all-injury crashes (pooled RR $0.78,95 \%$ CI 0.63 to 0.97 for studies with area control data; pooled RR $0.68,95 \%$ CI 0.61 to 0.77 for studies with daytime control data). When separated out, new street lighting produced a statistically significant reduction in both total injuries and fatal injuries compared to no lighting, and in total crashes for the studies with area control data.

None of the included studies reported usable data about changes in road traffic speed or perceived road user safety as a result of new or improved street lighting.

Results from this review suggest that street lighting may prevent road traffic crashes, injuries and fatalities in high income countries with well developed infrastructures (where all included studies took place). This is a particularly relevant finding in the UK, where an increasing number of local councils are looking towards turning off street lighting in certain areas in a move to reduce costs and carbon emissions. ${ }^{5}$ Further research should be focused on the effects of street lighting in LMICs and impacts on vulnerable road users (such as pedestrians), who are disproportionately affected.

This publication is the 17 th systematic review of a road safety intervention produced by the Cochrane Injuries Group. The full text of these reviews and systematic reviews on other injury prevention topics are available on the Cochrane Library (http://www.thecochranelibrary.com). For further information about the Cochrane Injuries Group, visit http://injuries. cochrane.org.

\section{F R Beyer, K Ker}

Correspondence to: Mrs F R Beyer, Institute of Health and Society, Newcastle University, 19-21 Claremont Place, Newcastle upon Tyne NE2 4AA, UK; fiona.beyer@ ncl.ac.uk

Competing interests: None.

Injury Prevention 2009;15:282. doi:10.1136/ip.2009.022251

\section{REFERENCES}

1. Peden M. World report on road traffic injury prevention. Geneva: World Health Organization, 2004

2. Beyer FR, Ker K. Street lighting for preventing road traffic injuries. Cochrane Database Syst Rev 2009;(1):CD004728.

3. Elvik R. Meta-analysis of evaluations of public lighting as accident countermeasure Transp Res Rec 1995;1485:112-23.

4. Vincent T. Streetlighting and accidents. Paper no. 17. Esso-Monash Workshop on Traffic Accident Evaluation. 15-17 Feb 1983.

5. BBC. Safety fears as the lights go out. 2008. http://tinyurl.com/9vmgw2 (accessed 26 Feb 2008). 